\title{
AGILE PRINCIPLES AND PRACTICES IN PROJECT MANAGEMENT
}

\author{
Elena MIRCEA \\ Bucharest University of Economic Studies, Romania \\ elenamircea94@gmail.com
}

\begin{abstract}
In the past 20 years the IT market has met a spectacular evolution. Nowadays complex technologies are used which can go beyond the power of the human brain. Daily business activities were automatized, and it is desired to eliminate the repetitive work. Our society has to constantly adapt to the new values and principles that govern the world and accept the changes by developing new skills. The aim of this paper is to define the agile techniques together with the most important project management principles, by combining them and obtaining new interactive ways of work. Spontaneous people and flexible processes lead to agile business, not by avoiding rules and regulations, but by creating new laws in scope of obtaining the highest results in a short period of time. In a world dominated by computers, people are trying to find ways of combining the human specific activities with the needs of the company.
\end{abstract}

Keywords: agile, project management, Scrum, simple practices

JEL classification: L86, M15, O3

DOI: $10.12948 / \mathrm{ie} 2019.05 .02$

\section{Introduction}

Software development has known various stages, but only in the '70s some general rules were established under a generic name. Over the years, in software development were implemented several models, starting with "Code and Fix" approach in which the team wrote the code and all the bugs were solved in the end. The application was delivered to the end customer and even the smallest changes were difficult to implement, as in some cases the whole program had to be modified. In the mid-1950s specific steps were defined, stages that had to be followed by each software development team: client requirements, design, development, integration, testing and deployment. This methodology became known as "Waterfall" model in 1970 and it is still used today in many projects. The principle behind the model is based on dividing the program in more phases and starting to work at the first one. After this is finished, the team can start to work on the next phase, but going backward for extra changes is very difficult, losing time and money. All the customer requirements are established from the beginning, so any modification comes with a high impact for the development team. Also, the customer involvement is quite limited during the development. In 10 years, another model was released, called "Spiral" and it is the first one that introduced terms like iterative and incremental. In the ' 90 s the idea of frequent feedback started to grow and an alternative to the Waterfall model appeared, "Rapid Application Development".

In 2001 the agile methodology was clearly defined by a group of developers, which understood the importance of learning from the previous iterations and from their own mistakes. In no more than 68 words they described the most important values of agile and pointed the principles which help obtaining high value software. The basic idea suggests setting on a second place the time-consuming processes and increasing the human interactions. [1]

"Agile" is mainly used in the IT industry, for defining a Project Management methodology, but sometimes it is wrongly believed that only in IT this model works. The agile practices can 
www.conferenceie.ase.ro

be used in every industry, from human resources to financial departments and even in the day by day life activities. The agile processes help the team to work after their own rules, improving the model based on the constant feedback. Each team member has the opportunity to get involved and expose his idea.

The special feature which distinguishes the agile software development from any other model is the way in which the product is delivered, after each step the customer can find new functionalities in the application. A flexible, dynamic and client focus product is provided to the end user.

\section{Agile Work - Rituals, Concepts, Instruments}

In literature [2], agile is considered more as a culture, rather than just a way of work, so several key concepts need to be known in order to be able to use the agile methods. First, all the team members are equals, positions are not clearly defined. Everyone can be a developer, tester, analyst at any point in time if this is needed. Scrum is the most popular agile method; daily activities are based on the past experiences and not only on theoretical aspects. In [3], Scrum is described as an iterative and incremental way of work.

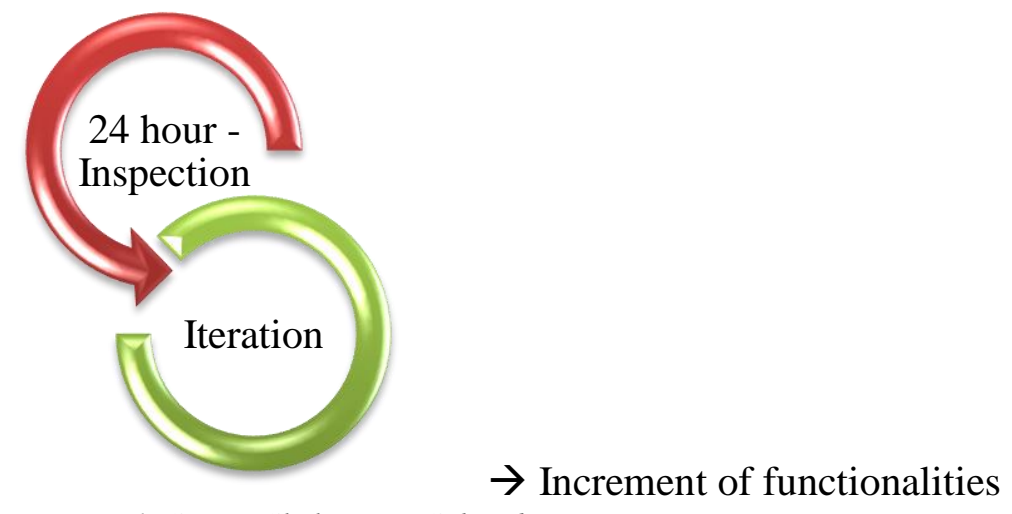

Task List $\rightarrow \quad$ Figure 1. Scrum Skeleton in Schwaber opinion

Agile teams are small and self-directed, the tasks are distributed taking into consideration the project needs. The following roles are defined:

- Stakeholder - financially affected by the result (client);

- Product Owner - the customer himself or someone who represents his needs and interests; each team has one PO which prioritize the tasks, takes care of the budget and knows all the functionalities that have to be implemented;

- Scrum Master - can be considered as a team leader, has very good agile skills and is the owner of the agile meetings

- Delivery Team - the main goal is to deliver on time, according to the agreed standards.

The team must deliver after each iteration, which is a fix period of time (2,3 or 4 weeks) called sprint in Scrum. Every two or three months a release is scheduled, and new functionalities are taken into production. Releases and sprint duration are set from the beginning can cannot be modified during an iteration.

Specific concepts are defined and without knowing them a new member cannot integrate in the team: [4]

- User Stories - customer requests, which will be used as task: As a user/customer, I want to <action> so that <benefit>. (Should be independent and valuable actions, testable and dimensioned for one sprint.);

- Story Points - unit measure for user story, can be considered an estimation of the work; 
- Product Backlog (Master Story List) - list containing all the user stories, owned by the PO. "As long as a product exists, the Product Backlog also exists" [3];

- Sprint Backlog - tasks which will be executed during one sprint.

Communication is a base element in project management, so as part of the agile methods, some rituals were defined, meetings that take place regularly: [4]

- Sprint Planning - defining user stories for the next sprint (Delivery Team and Scrum Master);

- Daily Stand-up - 15 minutes daily meeting, discussing what was done in the previous day, what will be done today and the impediments encountered (Delivery Team and Scrum Master);

- Sprint Review - team presents what was delivered over a sprint (Product Owner, Delivery Team and Scrum Master);

- Retrospective - lessons learned, meeting done after a sprint. (Delivery Team and Scrum Master).

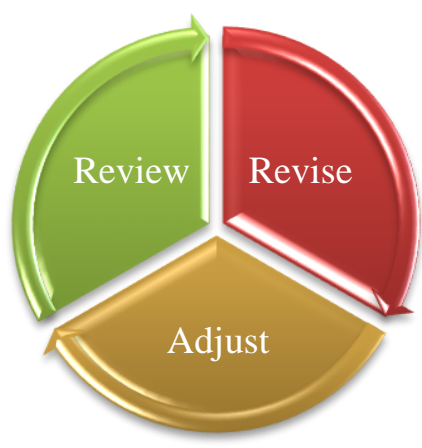

Figure 2. Lessons learned

As in management everything must be transformed into a number, KPIs which confirms if a project is profitable or not were defined also in agile, some metrics that calculates the performance of the teams. One of them is velocity, which refers to the number of story points that were delivered over a sprint. Another one is the Minimum Viable Product (MVP) which follows the minimum product that meets the client's expectations. Refers to everything that the program must have and not to what would be nice to have. Always the funnel of work must be prioritised and each member has to work at one task at a time, this way he will be concentrated over just one think.

Implementation of agile methods in an organization involves using some specific tools, that help teams visualize their work. [2] It is important to know if the team is distributed or not, since different approaches must be followed. It is compulsory to use burn down charts and agile boards (Kanban) when working agile, in order to see what needs to be done, the work in progress and what was already done. Each team can choose between the classic way, with sticky notes and panels on the walls or technology by using specific tools (Jira, Trello, Wrike etc.).

Distributed teams cannot be managed as locals, every team member must permanently see the agile table. Using paper sheets or internal tools in such cases will only ensure the failure of the project. Successfully distributed teams are conducted by some simple rules.[2] Remote colleagues do not have to be treated as if they were locals and it is valid also the other way around. Geographically speaking, if latitude is acceptable, as the team members are coming in the office at the same hour, longitude should not be accepted, or at least with no big differences. The company must invest in appropriate tools, but also should permit travels, it is recommended that the members of a team meet at least for one of the usual meetings per sprint. 
www.conferenceie.ase.ro

\section{Project Management Using Simple Agile Practices}

Agile model does not come with too many rules or restrictions, but it is recommended to follow the basic principles while working this way. In [2] this methodology was defined in an interesting way and were shown different approaches that can be followed without overwhelming the team. Agile is about simplicity and for any problem a simple solution should be found:

- When the team meets for the first time some general rules are set, about people behaviors, actions that are acceptable or not inside the team. These are written on sticky notes and placed in a visible place. This represents the social contact of the team and whoever breaks the rules has to speak in front of the team.

- The agile team must be constantly motivated, so the general atmosphere should be known. In the classical approaches, surveys are popular, in this case a simplified method is used, by having a jar and some smalls balls in two colors (e.g. black and white). Every morning the employee chooses a ball and puts it in the jar. At the end of the day, the manager can see if the team is happy or not by analyzing the jar and finding the predominant color.

Communication can be considered the base of project management, especially in agile. In order to collaborate, is crucial for the teams to freely express their ideas, but with respect for the others. The main questions in agile becomes "How can I do it better/faster?", every time the process must be improved by obtaining a better time or better quality of the product. Four human values were identified as being also the agile values, the ones that stay above of all the practices.

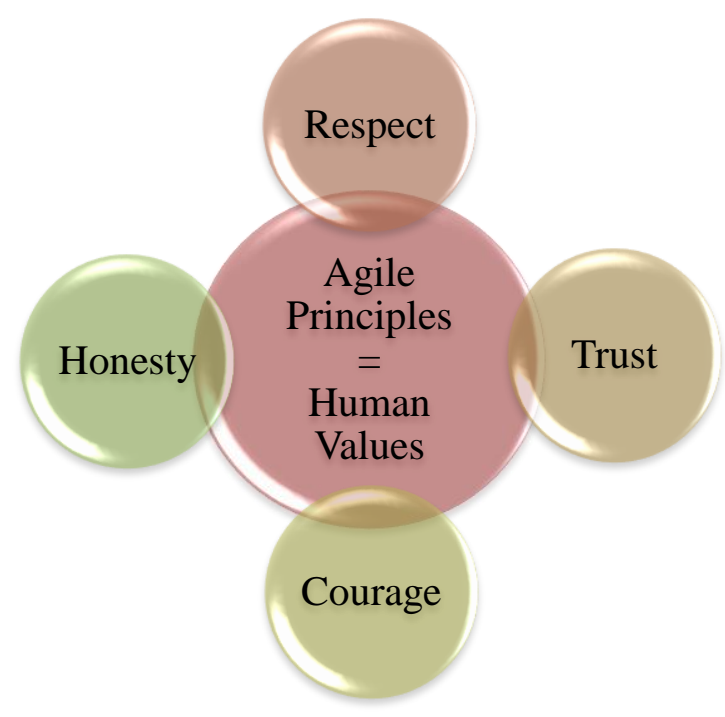

Figure 3. Agile Values according to [2]

Agile is a way of work based on a set of values and principles:

- Clearly define the outcome, focus on the result and less on organizing the team;

- Work and learn based on repetition - listen, iterate, learn and correct;

- Self-organized and small teams.

The consultant Patrick Lencioni described in [5] the five dysfunctions of a team by analyzing the dynamic inside of a group and gathering the common mistakes, offering solutions to improve team performance. Key concepts like trust and fear of conflict were disposed in a pyramidal form trying to emphases the management mistakes that are done in a company. Since sharing ideas is not encouraged during business meetings, people are afraid of commitments 
when things are not clear. According to Lencioni, because of these common faults, in the end, no results are obtained since no common purpose was agreed inside the team.

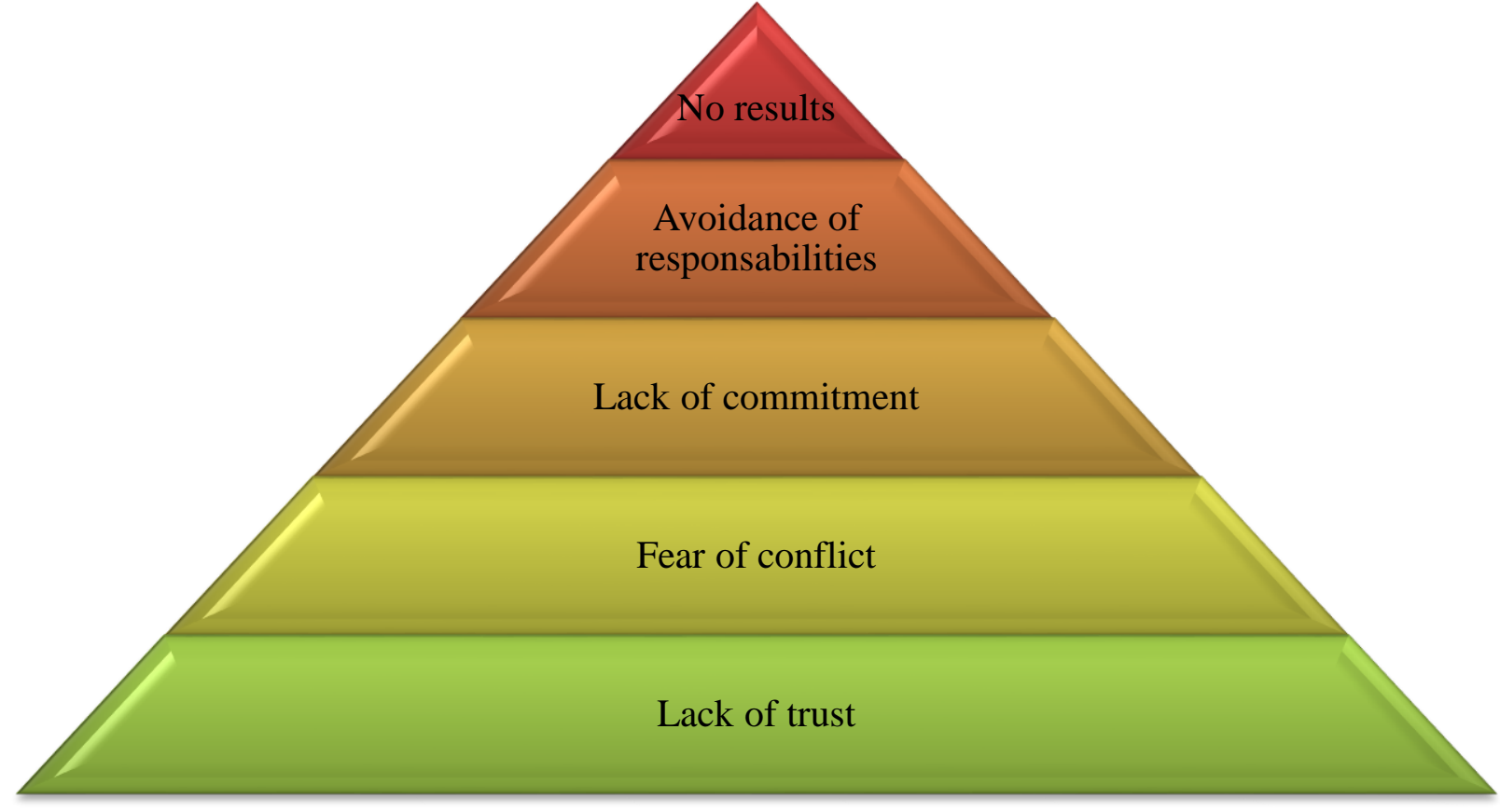

Figure 4. The five dysfunctions of a team

Agile processes and practices have to be followed and respected by the whole team and every step described in books must be implemented, every meeting has to take place. As Schwaber recommended in [3] changes in the processes are not allowed, especially in the beginning, when teams are learning how to manage the new roles and responsibilities. After years, for mature teams, when it is considered that the knowledge is strong enough, modifications can be done, and methods can be adapted depending on the needs. This method learning, called ShuHa-Ri (follow-break-transcend), is originally from Japan and became very popular in agile, describing the stages known by a team during the years. [6]

- "Shu" is the first phase, when the team members collaborate for the first time, they are just following the agile rules without ignoring any of the practices.

- "Ha" is the intermediate stage, some behaviors and habits are already formed and accepted by the whole team and if some more freedom is needed, small adjustments can be done to the standard processes.

- "Ri" describes a team that has many years of experience, each member can learn from his own experience and adapt the knowledge for the project. Even in this case, a very small change has to be discussed and agreed with the whole team.

Above all the rules and principles developed in agile, some myths were formed, and it is wrongly supposed that standard activities from the classical way of working do not exist here. Documentation exists in agile, the only difference is that it is not written in the beginning on the project, but only after all the things are clearly defined. As in this methodology, communication is over any written document. First, the team members, together with the customers review the product and then specifications can be documented. This way, rework is avoided, and the workflow approval is shortened. 
www.conferenceie.ase.ro

\section{Conclusions}

In conclusion, agile is a set of values, principles and practices that will change behaviors, redefining the way of work, by visualizing it as a new culture and less as overwhelming processes. When developing software using agile methods the team starts the iteration by evaluating what should be done, then selecting and transforming a potential functionality in the incrementation needed in the end.

In the end of this study, some limitations have to de mentioned, as the paper focuses on Scrum methodology. Since it is considered to be the most popular agile technique, the methods and tools used in Scrum are described together with specific metrics and rituals. But the agile practices are valid for all the agile teams, regardless the methods used.

It is wrongly believed that things are unorganized or unplanned in agile. Taking into consideration all the rituals that must be followed, all the tools that should be used, can be said that this methodology brings order and innovation for human resources more than any other method. Flexibility does not mean that the project is not organized, but only comes with some benefits for the employees by letting them propose new ideas and express their feelings. Changing roles is not similar with chaos, it means that everyone has the opportunity to learn different things and see the daily work from a different perspective.

Regardless the field of activity that defines a company, a good project manager should be characterized by openness, this is the reason why agile practices are not viable only for IT projects. Daily meetings or retrospectives are welcome in any office if the people want to improve the business. Of course, agile does not solve all the problems, but comes with solutions which can be applied in all the cases. As long as the people are willing to collaborate and communicate, the work environment becomes a place where every person can evolve by learning new skills and sharing knowledge.

\section{References}

[1] K. Beck, M. Beedle, A. von Bennekum, A. Cockburn, M.n Fowler, J. Grenning, J. Highsmith, A.Hunt, R.Jeffries, J. Kern, B. Marick, R. C. Martin, S. Mellor, K. Schwaber, J. Sutherland and D. Thomas, Manifesto for Agile Software Development, 2001.

[2] IBM Agile Explorer, "Think Academy," 2018 (last update). [Online]. Available: https://agile-ibm.mybluemix.net/courses/taste-of-agile/agile-principles-values-andbehaviors. [Accessed 23 November 2018].

[3] K. Schwaber, Agile Project Management with Scrum, Microsoft Press, 2004.

[4] M. Rubio, "An Agile Crash Course: Agile Project Management and Agile Delivery," 27 June 2018. [Online]. Available: https://learning.oreilly.com/home/.

[5] P. Lencioni, The Five Dysfunctions of a Team, John Wiley \& Sons, Inc., 2002.

[6] L. Goncalves, Organisational Mastery - The blueprint to create effective, efficient and highly rewarded executive leaders, evolution4All. 